\title{
Design and analysis of MEMS based capacitive pressure sensor for the detection of colon cancer
}

\author{
Syed Shameem, G.R.K. Prasad", G. Kalyan, H. Sri varun, P.S. Srinivas Babu \\ Micro Electronics Research Group, Department of ECE, Koneru. Lakshmaiah Education Foundation, \\ Vaddeswaram, Guntur, Andhra Pradesh, India-522502 \\ *Corresponding author E-mail: shameem9syed@gmail.com
}

\begin{abstract}
Emerging sciences have brought tremendous changes in the electronic world. One such revolutionary technology is MEMS. MEMS extend their implementation into many sectors like mechanical sensors, micro fluids, medical implants, bio-MEMS. In this paper, we discuss the design of a Micro Bio-sensor which senses the capacitance fluctuations materialized in it by various reactions of antigen and antibody. It is a cantilever based structure which is coated with a drug that is responsive to Carcino Embryonic Antigen (CEA), which is considered to be a first level indicator of colon cancer present in human body. The presence of this disease causing element in the blood initiates the antibody-antigen interactions which induces changes in the electrical parameters of the sensor device. These changes are utilized to determine the severity level of pathogens present in the patient's blood.
\end{abstract}

Keywords: Bio-MEMS, Capacitance, Cantilever, CEA, antibody-antigen reaction, Deflection of cantilever, Capacitance.

\section{Introduction}

MEMS are ultra small scale devices which are used to model electro mechanical systems. These devices are designed by fabrication and manufacturing at micro level. MEMS are small micro mechanized systems that normally are framed in small chips for various purposes [1]. These are structures based on silicon and are produced by engraving and micro manufacturing techniques. MEMS play an important role in the developing field of medicine and nanotechnology. MEMS are used to develop micro scale biosensors that will be used in many biological operations. It is also used to various commercial and industrial applications. Micro cantilevers have ability to show higher sensitivity [2-3]. Detecting mechanism of smaller scale cantilever biosensor varies as indicated by application [4-6]. The capacitive technique gives more sensitivity compared with mechanical stress and piezoresistive structure [7]. In this document, the elements of the biosensor are analytes, bio-element and transducer. An analyte interaction with the bio-element that makes an effect that the transducer that becomes an electrical signal. The bio-element can be a protein, enzyme, an antigen or an antibody, etc. Biosensor has two functions i) change from bio-molecular to electrical recognition signal, ii) Bio-element that identifies analyte for a maximum performance cantilever.

\section{Problem description}

In there cent times we tend to see that there's a zoom of superfluity of diseases. Most of those sickness inflicting agents manifest within the target bodies very quickly and disrupt the important functioning of the body proving it to be fatal to the human. So there's a high need to devise a sensor which might find the presence of sickness inflicting pathogens within the samples so that emergency medication can be given to the patient and make the condition stable. The ability to perform diagnosis at the bedside or at the point-of-care is very important in health care, particularly in developing countries wherever access to centralized hospitals is restricted and prohibitively costly. To present the result, point-of-care diagnostic bio MEMS are developed to take saliva, blood, or urine. Blood may be a quite common biological sample as it cycles through the body each couple of minutes and its contents will indicate several aspects of health. POC gadgets incorporate microchannels, microsensors, and so forth, which have been connected to a few, scaled down POC items. Probably the most normally utilized POC gadgets, for example, blood glucose estimations and pregnancy tests, show that in-home testing by the patient is popular.

\section{Design methodology}

Selectivity and specificity are the principle confinements for the execution of biosensors which depends on the structure and methodology used in the sensing process. The proposed structure of this capacitance based bio sensor is designed using COMSOL Multiphysics tool in the form of a two beam composition among which one is a micro cantilever and the other being a fixed one. This setup acts a capacitor with two metal plates separated by a dielectric medium. A Cantilever beam is a free end sort of hanging structure, which can without much of a stretch, be occupied with mass load. If there should be an occurrence of recognizing the diversion or distortion of the cantilevered structure, it relies upon 
the connected weight yet in addition on numerous other geometric shapes parameters. The diversion increments with the length of the cantilever and decrements with its thickness. Additionally, in Changing the cantilever redirection also relies upon both activating voltage and geometric parameters. Biosensors utilize optical discovery strategy to gauge surface anxiety initiated redirections in a micro cantilever. At the point when the objective particles join their functionalized surface, the dispersion of surface pressure at first glance changes causing redirections in the cantilever amid the adsorption of target particles in the functionalized cantilever surface, biochemical responses happen that lessen the free vitality of the cantilevered surface. The lessening in the free vitality of one side of the cantilever is adjusted with increment in the voltage vitality of the opposite side delivering redirection in the cantilever. Biochips with mechanical location frameworks normally utilize light emissions micro cantilever materials (eg, Au-Si) as detecting components. The $\mathrm{Au}$ side is generally layered with a specific collector. After authoritative of the analyte to the receptor, the surface of the receptor is extended or alleviated. This causes the micro cantilever to deviate, ordinarily in nanometres, which can be measured utilizing optical systems. The deviation is relative to the analyte level of concentration. The idea has been utilized as a part of the discovery of specific infections, for example, growth and the identification of particular synthetic and organic fighting specialists.

\subsection{Antibody-Antigen Reactions}

The antigens are little intruders that enter the body and enact the invulnerable framework. Every antigen can make contamination to the body. An element named Carcino Embryonic Antigen (CEA) is considered to be a first level indicator of colon cancer present in human body. CEA depicts an arrangement of exceedingly related glycoprotein associated with cell grip. CEA regularly happens in the gastrointestinal tissue amid fetal improvement, however creation stops before birth. In this way, CEA is typically present just at low levels in the blood of solid grown-ups. Be that as it may, serum levels are raised in a few sorts of malignancy, which implies that it can be utilized as a tumor marker in clinical trials Serum levels can likewise be lifted in overwhelming smokers. The various levels of antigens found in adults classified as smokers and non-smokers is given in Table 1.

Table 1: Levels of CEA present in adults classified as smokers and non-smokers

\begin{tabular}{|c|c|c|}
\hline Gender & Smoker(ng/ml) & Non-Smoker(ng/ml) \\
\hline Male & $2.27+/-(1.67)$ & $2.08+/(0.91)$ \\
\hline Female & $3.15+/-(1.51)$ & $1.35+/-(0.67)$ \\
\hline
\end{tabular}

The area of an antigen that connects with a counter acting agent is called an epitope. The antibody that detects the CEA antigen is
Beacizumab. It focuses on a tumor cell protein called vascular endothelial development factor (VEGF). This protein causes tumor to create veins, so they can get nourishment and oxygen from the blood. All growths require a blood supply to survive and develop. Bevacizumab hinders this protein and prevents malignancy from developing in the veins, so cannot develop.

In the proposed structure, for the antibody-antigen reaction to happen, a cavity is dug on the free end of the upper beam which acts as a canal for the sample deposit.The canal structure is coated with a layer of antibody that is responsive to antigen CEA. Whenever the organic sample containing the CEA antigen is dropped onto the device sensor, it is channeled through the cavity provided in the sensor. As the cavity is coated with the antibody, due to the reactions occurring between antigen-antibody pair, the antigen particles get adhered to the surface of the antibody in the cavity and the rest of the sample passes down the channel as it slantingly arranged. As a result of these particles getting bonded due antigen-antibody reactions, the outcome of the reaction at the onset of equilibrium is that they contribute to some mass on the device structure. This amount of mass deposited on the device kick starts the sensing action the device based on mass-sensing principle.

Calculation of capacitance for displacements caused due to applied force:

Capacitance between two beams of cantilever setup can be given as

$\mathrm{C}=\mathrm{A} \varepsilon_{0} \varepsilon_{\mathrm{r}} / \mathrm{d}$

Where

$\varepsilon_{0}=$ permittivity of free space $=8.854 \times 10 \mathrm{e}-12$

$\varepsilon_{\mathrm{r}}=$ relative permittivity $=1$

$\mathrm{A}=$ Area of the plates(beam)

=length of beam $x$ width of beam $=100 \mu \mathrm{m} \times 20 \mu \mathrm{m}=2000 \mathrm{x} 10 \mathrm{e}-$ $12 \mathrm{~m}^{2}$

So capacitance $\mathrm{C}$ can be given as

$\mathrm{C}=\left(2000 \times 10 \mathrm{e}-12 \mathrm{~m}^{2} \times 8.854 \times 10 \mathrm{e}-12\right) / \mathrm{d}$

Where' $d$ ' determines the displacement caused due to deflection in beam, as a result of force applied by antigen binding.

Let us assume 1ng of CEA antigen is binded to the surface

Then, 1 nanogram contributes to $9.80665 \mathrm{e}-11 \mathrm{~Pa}$ of pressure on the surface

Weight of CEA antigen molecule is $=180000 \mathrm{Da}=2.98897006 \mathrm{e}-$ 10ng $=3$ ng

So if $\tilde{3} \times 10 \mathrm{e} 11 \mathrm{ng}$ molecules of CEA are assumed to binded then

Pressure applied $=3 \times 10 \mathrm{e} 11 \times 9.80665 \mathrm{e}-11 \mathrm{~Pa}=29.41995 \mathrm{~Pa}=$ $30 \mathrm{~Pa}$

So we apply loads ranging from $10 \mathrm{~Pa}$ to $40 \mathrm{~Pa}$ and determine the capacitance variations for applied pressure.

\section{Results}

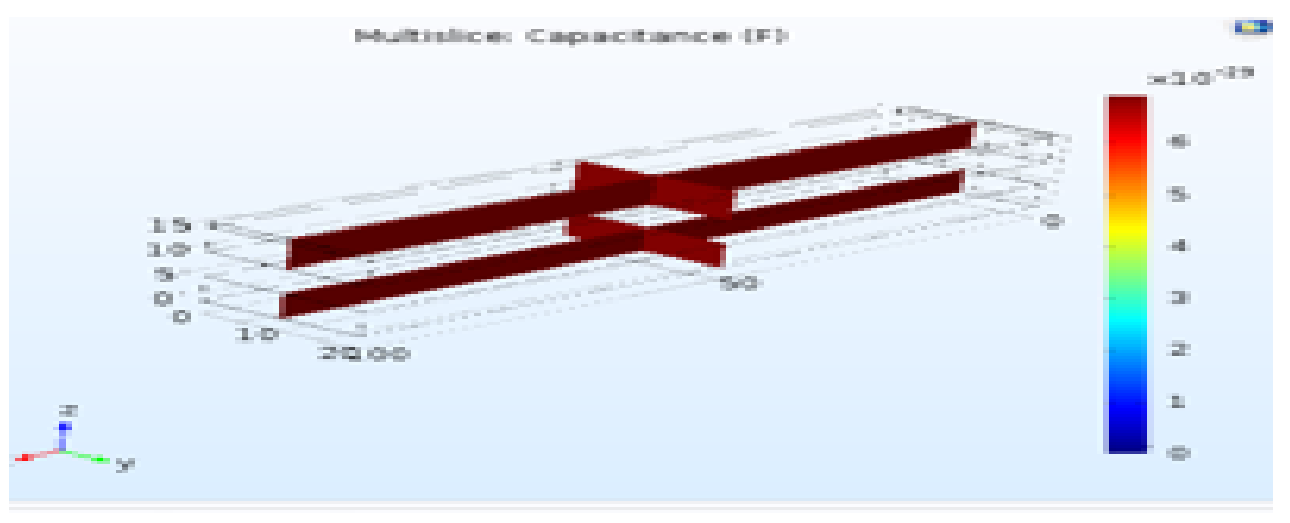

Fig. 1: Two beam cantilever Multi slice structure for capacitance variations 
The two beam cantilever structure in figure 1 is analyzed for capacitance variations along the multi slice cut so that uniform changes in the electrical property of the material is observed.



Fig. 2: Capacitance variations for different voltages applied to the two beam structure

This plot in figure 2 demonstrates the changes in the beam capacitance for varying voltages. Here the voltage is analogous to the load that is added onto the antibody after the antigen gets binded to the surface. This induces voltage variations causing the deflections in beam. As a result the capacitance changes linearly with increasing voltages.

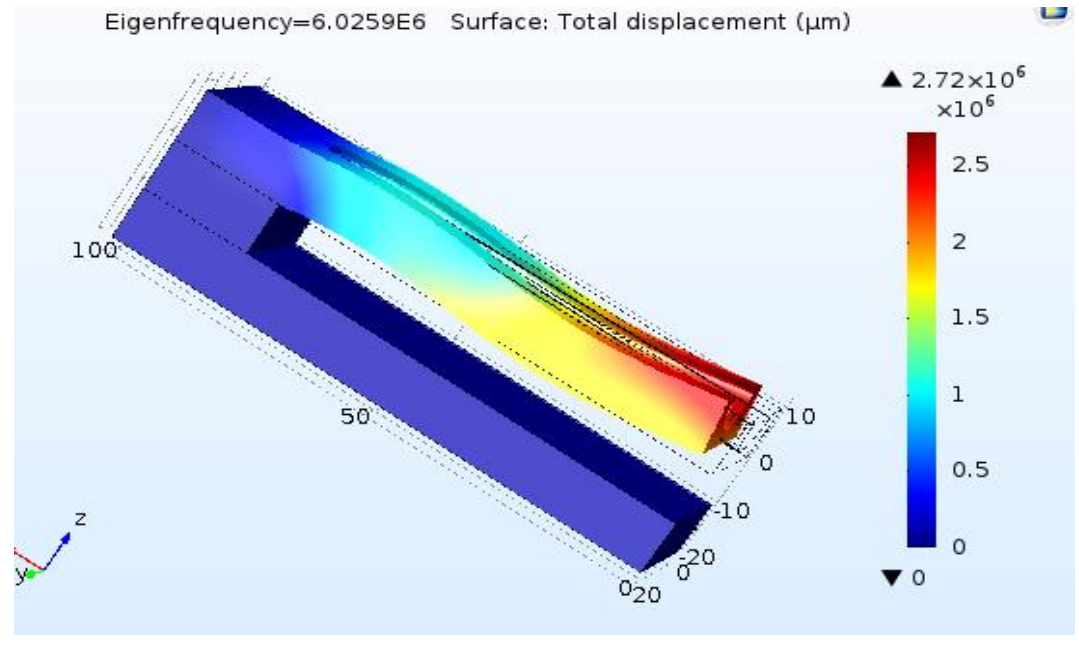

Fig. 3: Eigen Frequency vs Displacement variations of beam at frequency at $\quad 6.0259 \mathrm{E} 6$

Eigenfrequency $=4.4371 \mathrm{E} 6$ Surface: Total displacement $(\mu \mathrm{m})$

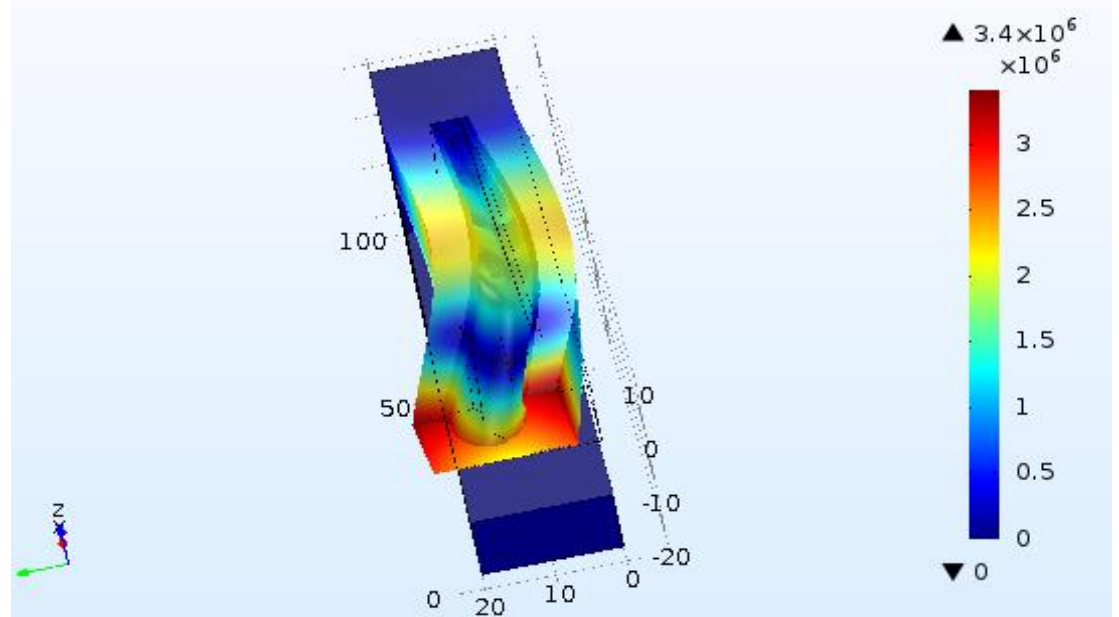

Fig. 4: Eigen Frequency vs Displacement variations of beam at frequency at 4.4871E6 




Fig. 5: Eigen Frequency vs Displacement variations of beam at frequency at 2.7928E6 Eigenfrequency $=9.688 \mathrm{E} 5$ Surface: Total displacement $(\mu \mathrm{m})$

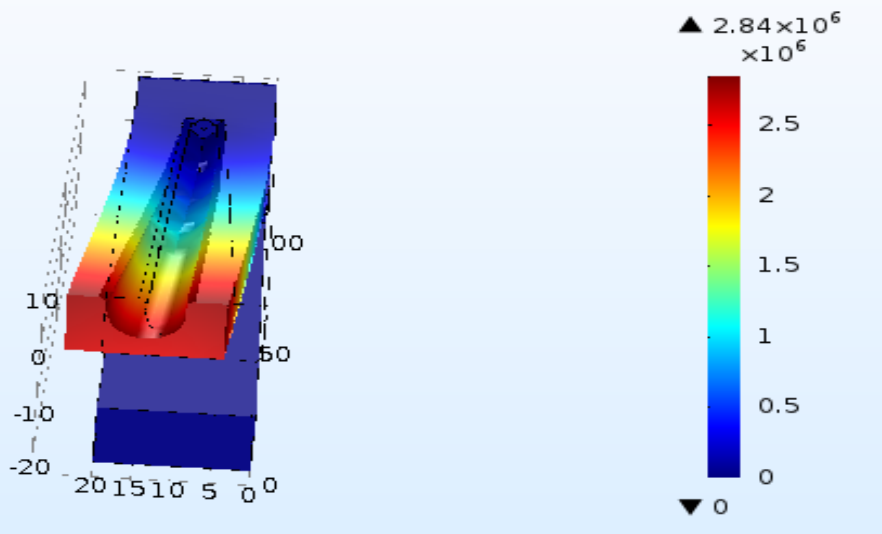

Fig. 6: Eigen Frequency vs Displacement variations of beam at frequency at 9.688E5

Eigenfrequency $=5.0605 E 5$ Surface: Total displacement $(\mu \mathrm{m})$



Fig. 7: Eigen Frequency vs Displacement variations of beam at frequency at 5.0605E5

Eigenfrequency $=2.2878 \mathrm{E} 6$ Surface: Total displacement $(\mu \mathrm{m})$

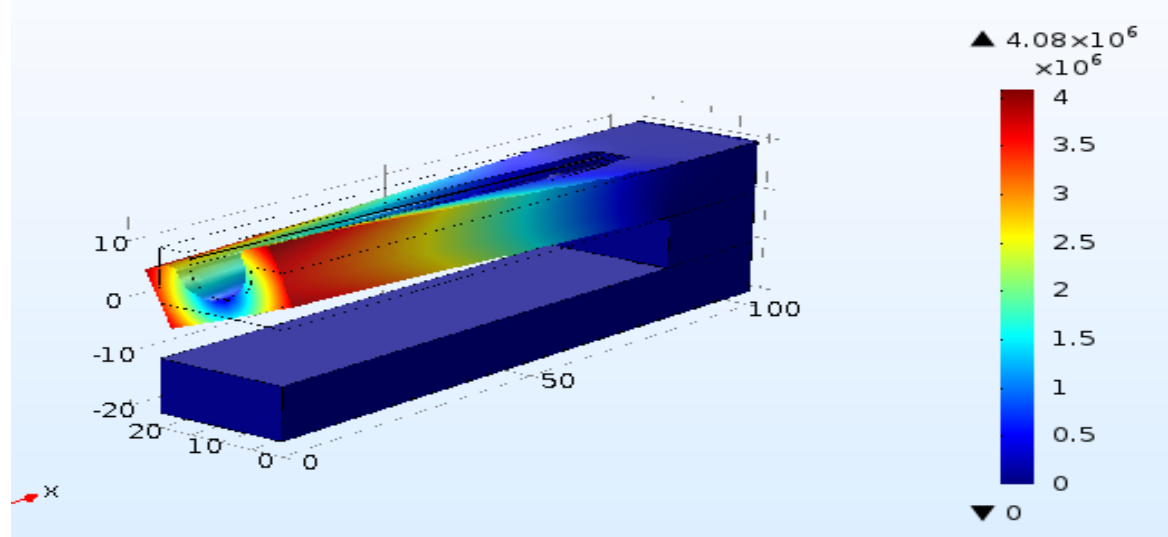

Fig. 8: Eigen Frequency vs Displacement variations of beam at frequency at 2.2878E6 
The two beam structure with antibody cavity is analyzed for various Eigen frequencies and the displacement caused due to the deflections of the beam is recorded at each of the frequencies

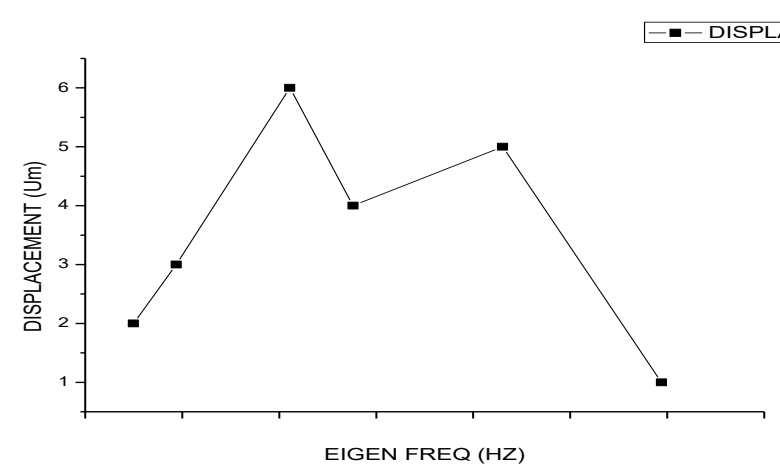

Fig. 9: Beam Displacement variations in response to eigen frequencies for applied force of 10Pa

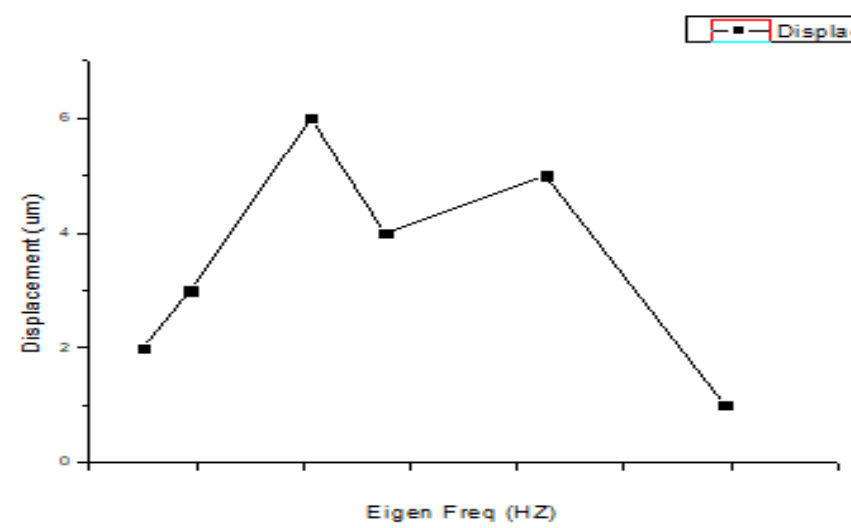

Fig. 10: Beam Displacement variations in response to eigen frequencies for applied force of $20 \mathrm{~Pa}$

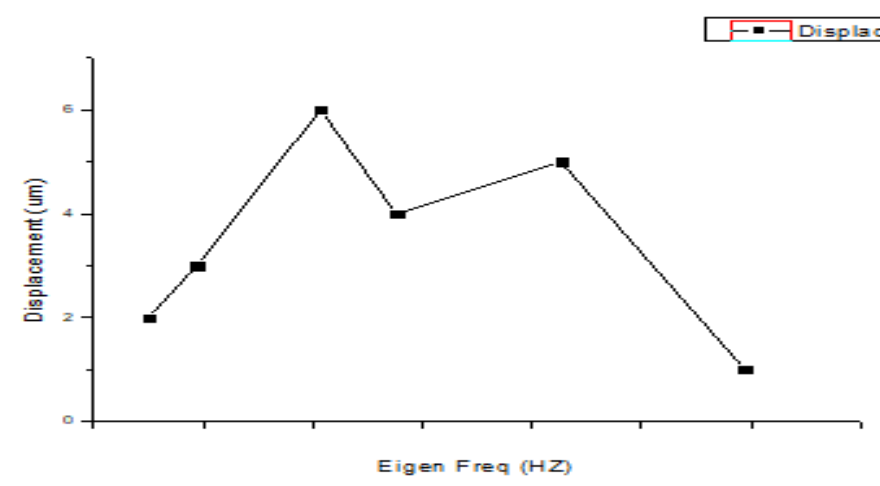

Fig. 11: Beam Displacement variations in response to Eigen frequencies for applied force of 30Pa



Fig. 12: Beam Displacement variations in response to eigen frequencies for applied force of $40 \mathrm{~Pa}$ 
The two beam cavity structure in Figure 12 is analyzed for varying set of pressures ranging from $10 \mathrm{~Pa}$ to $40 \mathrm{~Pa}$ and analyzed for displacement variations. The force applied is analogous to the load that is formed due to the antigen binding on the antibody surface.
This load causes deflections in the beam, thereby causing changes in the beam displacement. This change in displacement between the two beams causes change in capacitance given by formula $\mathrm{C}=\mathrm{A} \varepsilon_{0} \varepsilon_{\mathrm{r}} / \mathrm{d}$

Table 2: Capacitance variations due to displacements caused by various loads

\begin{tabular}{|c|c|c|}
\hline Pressure applied(Pa) & Beam displacement $(\mathrm{m})$ & Capacitance(F) \\
\hline 10 & $3.39^{*} 10^{\wedge} 6$ & $5.22^{*} 10^{\wedge}-15$ \\
\hline 10 & $3.41^{*} 10^{\wedge} 6$ & $5.19^{*} 10^{\wedge}-15$ \\
\hline 10 & $4.92^{*} 10^{\wedge} 6$ & $3.59^{*} 10^{\wedge}-15$ \\
\hline 10 & $3.63^{*} 10^{\wedge} 6$ & $4.87^{*} 10^{\wedge}-15$ \\
\hline 10 & $4.07^{*} 10^{\wedge} 6$ & $4.35^{*} 10^{\wedge}-15$ \\
\hline 20 & $3.06^{*} 10^{\wedge} 6$ & $5.78^{*} 10^{\wedge}-15$ \\
\hline 30 & $5.43^{*} 10^{\wedge} 6$ & $3.26^{*} 10^{\wedge}-15$ \\
\hline 30 & $4.28^{*} 10^{\wedge} 6$ & $4.13^{*} 10^{\wedge}-15$ \\
\hline 40 & $2.72^{*} 10^{\wedge} 6$ & $6.51^{*} 10^{\wedge}-15$ \\
\hline 40 & $3.15^{*} 10^{\wedge} 6$ & $5.62^{*} 10^{\wedge}-15$ \\
\hline
\end{tabular}

1

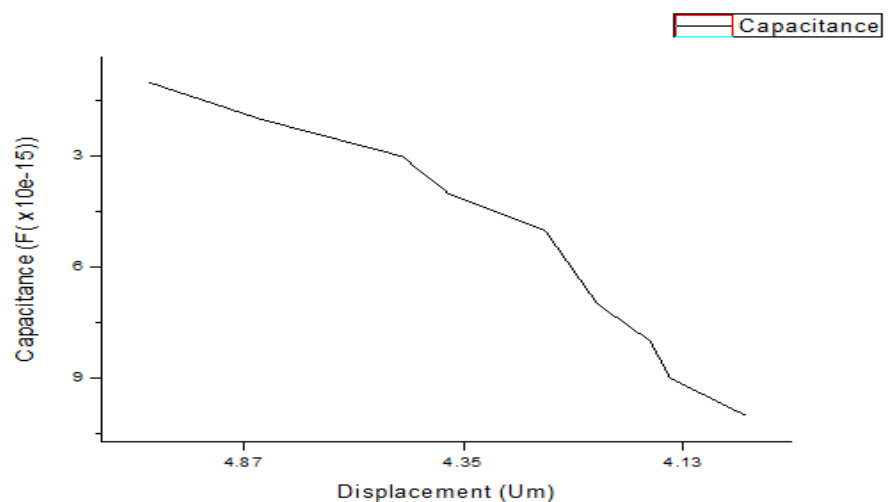

Fig. 13: Variations of capacitance of two beam cavity structures for varying displacements caused due to different loads

For the two beam cantilever setup with a conical cavity, various loads are applied which are analogous to the force caused due to antigen binding the surface. The force causes beam to deflect thereby varying the displacement between two beams, this induces a change in the capacitance of the cantilever setup for varying displacements. It is observed from table 2 and figure 13 that as the force is applied, more the displacement between beams, lesser is the capacitance induced in the structure.

\section{Conclusion}

In this work, we have first designed a simple two beam cantilever structure which was coated with a gold antibody. After performing eigen frequency analysis on it, the structure was analyzed for capacitance variations for varying voltages. Later we designed a cantilever setup with a conical cavity which can be used to realize a bio sensor that is responsive to organic samples. Mathematical analysis was performed so as to determine the density of drug that needs to be used to design the material. Then a new material resembling the drug's electrical and mechanical properties was designed using COMSOL and coated on the conical cavity. Later this cavity based cantilever setup was analyzed for various loads applied and corresponding changes in displacement of beams due to deflections caused. The antigen binding contributes to the deflections in the beam thereby causing the capacitance of the cantilever setup to change. Under applied load, as the displacement between two beams increases, the capacitance induced in the cavity based cantilever setup decreases. So this capacitance can be used as a measure to detect the amount of antigen (CEA) that is binded, which can be used as a first level indicator of the cancer disease.

\section{Acknowledgements}

The authors would like to thank NPMASS scheme for establishing national MEMS Design center in AP which is supported by IISC, Bangalore for providing the necessary design facilities.

\section{References}

[1] D. Klaitabtim and A. Tuantranont, "Design consideration and finite element modeling of MEMS cantilever for nanobiosensor applications," Procs. of 2005 5th IEEE Conference on Nanotechnology, Nagoya, Japan, July 2005

[2] V. Jain, S. Verma, "Design and analysis of MEMS piezoresistive three layers microcantilever-based sensor for biosensing applications," International Journal of Innovative Technology and Exploring Engineering, Volume-2, Issue-5, April 2013.

[3] P. G. Gopinath, V. R. Anitha, S. Aruna Mastani, "Microcantilever based biosensor for disease detection applications," Journal of Medical and Bioengineering, Vol. 4, No.4, August 2015.

[4] Y. Lu, V. Chivukula, M. Wang and H. F. Ji, "Simulation and fabrication of Si02-based piezoresistive microbridges for chemlbiosensors," J Micromech. Microeng, vol. 16,692698,2006

[5] S. Dhakane, W. V. Patil, "Piezo-resistive method for tuberculosis detection using microcantilever biosensor," Microsyst Technol, 2013.

[6] B. Bais, R. A. Rahim and B. Y. Majlis, "Finite element and system level analyses of piezoresistive microcantilever for biosensing

[7] Applications", Australian Journal of Basic and Applied Sciences, vol.5, No.12, pp. 1038-1046,2011

[8] Srinivasa Rao K, Sateesh Jasti, "Design and Analysis of MEMS based Bio sensor for TB detection, ICEECCOT, 2016.

[9] Dr. Seetaiah Kilaru, Hari Kishore K, Sravani T, Anvesh Chowdary L, Balaji $\mathrm{T}$ "Review and Analysis of Promising 
Technologies with Respect to fifth Generation Networks", 2014 First International Conference on Networks \& Soft Computing, ISSN:978-1-4799-3486-7/14,pp.270-273,August2014.

[10] Meka Bharadwaj, Hari Kishore "Enhanced Launch-Off-Capture Testing Using BIST Designs" Journal of Engineering and Applied Sciences, ISSN No: 1816-949X, Vol No.12, Issue No.3, page: 636-643, April 2017.

[11] N Bala Dastagiri, Kakarla Hari Kishore "Reduction of Kickback Noise in Latched Comparators for Cardiac IMDs" Indian Journal of Science and Technology, ISSN No: 09746846, Vol No.9, Issue No.43, Page: 1-6, November 2016.

[12] A Murali, K Hari Kishore, D Venkat Reddy "Integrating FPGAs with Trigger Circuitry Core System Insertions for Observability in Debugging Process" Journal of Engineering and Applied Sciences, ISSN No: 1816-949X, Vol No.11, Issue No.12, page: 2643-2650, December 2016.

[13] Mahesh Mudavath, K Hari Kishore "Design of CMOS RF Front-End of Low Noise Amplifier for LTE System Applications Integrating FPGAs" Asian Journal of Information Technology, ISSN No: 1682-3915, Vol No.15, Issue No.20, page: 4040-4047, December 2016.

[14] P Bala Gopal, K Hari Kishore, B.Praveen Kittu "An FPGA Implementation of On Chip UART Testing with BIST Techniques", International Journal of Applied Engineering Research, ISSN 0973-4562, Volume 10, Number 14 , pp. 34047-34051, August 2015.

[15] S Nazeer Hussain, K Hari Kishore "Computational Optimization of Placement and Routing using Genetic Algorithm" Indian Journal of Science and Technology, ISSN No: 0974-6846, Vol No.9, Issue No.47, page: 1-4, December 2016.

[16] N Bala Gopal, K Hari Kishore "Analysis of Low Power Low Kickback Noise in Dynamic Comparators in Pacemakers" Indian Journal of Science and Technology, ISSN No: 09746846, Vol No.9, Issue No.44, page: 1-4, November 2016.

[17] S.V.Manikanthan and K.Baskaran "Low Cost VLSI Design Implementation of Sorting Network for ACSFD in Wireless Sensor Network", CiiT International Journal of Programmable Device Circuits and Systems,Print: ISSN 0974 - 973X \& Online: ISSN 0974 - 9624, Issue : November 2011, PDCS112011008.

[18] Rajesh, M., and J. M. Gnanasekar. \&quot;GCCover Heterogeneous Wireless Ad hoc Networks.\&quot;Journal of Chemical and Pharmaceutical Sciences (2015): 195-200.

[19] T.Padmapriya, Ms. N. Dhivya, Ms U. Udhayamathi, "Minimizing Communication Cost In Wireless Sensor Networks To Avoid Packet Retransmission", International Innovative Research Journal of Engineering and Technology, Vol. 2, Special Issue, pp. 38-42. 